\title{
Stiftungen: Ein Weg der Wissenschaftsförderung
}

Foundations: One Way to Support Science

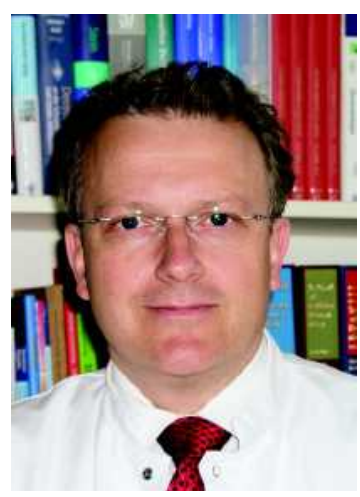

Prof. Dr. med. Dr. rer. nat. Christoph C. Geilen
Stiftungsneugründungen haben in den vergangenen Jahren stark zugenommen und immer häufiger gehen Privatpersonen, Institutionen oder Firmen diesen Weg, um ihre Vorstellungen und Interessen langfristig umzusetzen und zu fördern. In den Anfängen konzentrierte sich das Stiftungswesen in Deutschland auf die Schaffung von Spitälern und den Aufbau eines Fürsorgesystems für Arme und Bedürftige. So datieren die ältesten, heute noch bestehenden deutschen Stiftungen in das zwölfte Jahrhundert, in die Zeit Barbarossas, Friedrich I. (1152 - 1190). Neben der Unterstützung karitativer Zwecke ist die Kulturförderung seit jeher ein Schwerpunkt privaten Mäzenatentums, dessen Urvater und Namensgeber, Maecenas ( 70 v. Chr. -8 v. Chr.) zu Zeiten Kaiser Augustus Dichter wie Horaz und Vergil förderte.

Der Stiftergedanke hat in den vergangenen Jahren, auch weil sich die gesetzlichen Rahmenbedingungen durch das „Gesetz zur weiteren Stärkung des bürgerlichen Engagements“ erheblich verbessert haben, immer mehr durchgesetzt. Allein 2007 kamen in Deutschland 1134 neue Stiftungen hinzu, sodass Deutschland mit seinen über 15500 Stiftungen in Europa unter den ersten Plätzen zu finden ist. Die thematische Verteilung der Förderziele spiegelt die historische Entwicklung des Stiftungswesens wider: 34\% der Stiftungen verfolgen soziale Zwecke, $19 \%$ fördern Kunst und Kultur und ca. 13\% haben die Unterstützung von Wissenschaft und Forschung im Fokus. Diese Reihenfolge ist auch dadurch erklärbar, dass Wissenschaftsfinanzierung immer noch als reine Domäne des Staates empfunden wird. Dies ist in anderen Staaten, wie den USA oder Frankreich, anders, so sind in Frankreich beispielsweise $50 \%$ der Stiftungsausgaben der Medizinforschung gewidmet.

In der Dermatologie haben sich in den vergangenen Jahren eine Reihe von Stiftungen etabliert, die sich die Förderung des wissenschaftlichen Nachwuchses und der dermatologisch-relevanten Forschung auf die Fahne geschrieben haben. Die erste Stiftung auf diesem Gebiet war die Marchionini-Stiftung, 1999 folgte die Gründung der Berliner Stiftung für Dermatologie, über deren diesjähriges, wissenschaftliches Symposium in dieser Ausgabe berichtet wird. Schließlich kam die Deutsche Stiftung für Dermatologie (2005), die durch die Deutsche Dermatologische Gesellschaft gegründet wurde, hinzu und das Deutsche Hautforschungszentrum folgte als Stiftung im Jahre 2007. Insofern ist die Dermatologie in Deutschland auch in dieser Hinsicht für die Zukunft gut aufgestellt und kann, unabhängig von staatlicher Forschungsförderung und der direkten Unterstützung durch die pharmazeutische Industrie, Forschungsvorhaben und den wissenschaftlichen Nachwuchs fördern.

Stiftungen sind eine Investition in die Zukunft. Um diese Aufgabe auch weiterhin und in verstärktem Maße gerecht zu werden, bedarf es der Stärkung ihres Stiftungskapitals. Spenden und Zustiftungen sind hier eine sehr gute Möglichkeit, dieses Engagement aktiv zu unterstützen.
Bibliografie

DOI $10.1055 / \mathrm{s}-2008-1077549$

Akt Dermatol 2008; 34; 293

(c) Georg Thieme Verlag KG .

Stuttgart · New York

ISSN 0340-2541

Korrespondenzadresse

Prof. Dr. med. Dr. rer. nat. Christoph C. Geilen

Stellvertretender Geschäfts-

führer der Berliner Stiftung

für Dermatologie

CCG Consulting Berlin $\mathrm{GmbH}$

Kurfürstendamm 21

10719 Berlin

Christoph.Geilen@

ccg-consulting-berlin.de

www.ccg-berlin.de 